\begin{tabular}{|c|l|}
\hline Title & Management of Renal A ngiomyolipomas A ssociated With Tuberous Sclerosis Complex \\
\hline Author(s) & Harabay ashi, Toru; Shinohara, Nobuo; Katano, Hidenori; Nonomura, Katsuy a; Shimizu, Tadashi; Koyanagi, Tomohiko \\
\hline Citation & $\begin{array}{l}\text { The Journal of Urology, 171(1), 102-105 } \\
\text { https://doi.org/10.1097/01.ju.0000100100.36354.61 }\end{array}$ \\
\hline Issue Date & 200401 \\
\hline Doc URL & http://hdl.handle.net/2115/30261 \\
\hline Type & article (author version) \\
\hline File Information & Jou171-1.pdf \\
\hline
\end{tabular}

Instructions for use 


\section{MANAGEMENT OF RENAL ANGIOMYOLIPOMAS ASSOCIATED WITH TUBEROUS SCLEROSIS COMPLEX.}

Toru Harabayashi, Nobuo Shinohara, Hidenori Katano, Katsuya Nonomura, Tadashi Shimizu and Tomohiko Koyanagi.

Department of Renal and Genitourinary Surgery and Diagnostic Radiology, Graduate School of Medicine, Hokkaido University, Sapporo.

Kita-15, Nishi-7, Kita-ku, Sapporo 060-8638, JAPAN.

Running title: Management of renal AMLs in TSC.

Text pages 11, Table 1, Figures 2.

Correspondence: Toru Harabayashi, M.D., Department of Renal and Genito-Urinary Surgery, Graduate School of Medicine, Hokkaido University, Kita-15, Nishi-7, Kita-ku, Sapporo 060-8638, Japan.

Phone: 81-11-706-5966, Fax: 81-11-706-7853. E-mail: harachan@med.hokudai.ac.jp.

Keywords: angiomyolipoma, tuberous scelerosis, embolization, observation. 


\section{ABSTRACT}

Introduction: Intensive management is generally recommended for angiomyolipomas associated with tuberous sclerosis complex (TSC), which are known to have a more aggressive nature than sporadic tumors. The purpose of this study was to evaluate the management of these tumors.

Patients and methods: Adult TSC patients (6 males and 6 females, mean age 28.8 years) with angiomyolipomas treated from 1984 to 2000 were retrospectively analyzed. All patients had bilateral multiple tumors (mean size $6.6 \mathrm{~cm}$ ). Lesions were classified as small $(<4 \mathrm{~cm}$, $\mathrm{n}=10)$, medium ( $4-10 \mathrm{~cm}, \mathrm{n}=6)$ or large $(>=10 \mathrm{~cm}, \mathrm{n}=8)$ based on the size. Computed tomography was annually repeated for followup observation and semiannually after embolization.

Results: Intervention was required in 20\%, 50\%, 100\% of kidneys with small, medium and large tumors, respectively. Among 14 kidneys followed by observation, 8 tumors grew and 4 required intervention. No patient developed uncontrollable hemorrhage. While some tumors showed remarkable growth in the third decade, growth speed did not exceed $1.5 \mathrm{~cm}$ per year. Embolization was performed in 11 kidneys and tumors shrank in 10 of them. The mean reduction rate of renal function was $9 \%$ in the patients treated with embolization, though one patient experienced renal atrophy. With a mean followup of 60 months, all patients but 1 are alive without renal failure.

Conclusions: Although asymptomatic angiomyolipomas associated with TSC gradually grow, severe hemorrhage is rare and most tumors can be conservatively managed by annual computed tomography. Embolization is the first choice of intervention but should be reserved until symptoms develop. 


\section{INTRODUCTION}

Tuberous sclerosis complex (TSC) is an autosomal dominant disorder transmitted by 2 genes: TSC1 and TSC2. The incidence is estimated approximately to be 1:10,000 births; however, 65 to $85 \%$ of the cases arise via spontaneous mutation. ${ }^{1} \quad$ It is characterized by seizures, mental retardation and hamartomatous lesions, including facial angiofibroma, subependymal giant cell astrocytoma, cardiac rhabdomyoma and renal angiomyolipoma (AML). Although traditionally recognized as a neurologic and dermatologic disorder, renal disease is a frequent manifestation of TSC and the leading cause of death in adults. ${ }^{2}$

Renal cell carcinoma can develop in TSC patients at a younger age than in the general population. Renal cysts can cause renal failure from progressive compression of functional parenchyma. The commonest manifestation in the kidney is AML. The AMLs associated with TSC usually show multiple, bilateral and symptomatic natures compared with sporadic AMLs. Some authors have recommended intensive intervention for patients with these tumors, and others have reported that all of these tumors do not necessarily require intervention. ${ }^{3,4}$ In this study, we retrospectively evaluate the treatment outcome of AMLs associated with TSC and discuss the treatment strategy for these tumors.

\section{PATIENTS AND METHODS}

From 1984 to 2000, 14 adult TSC patients were referred to our department. After excluding 1 patient with renal cell carcinoma and 1 patient who refused treatment, 12 patients with renal AMLs were reviewed. From the chart review, variables including clinical symptoms, tumor size, renal function and treatment were identified, and followup information was obtained from the chart or through correspondence with the referring physician. All patients had clinical diagnoses of TSC, as defined by the 1998 Tuberous Sclerosis Complex Consensus Conference. ${ }^{5}$ In all patients, the diagnosis of AML was 
made due to its unique appearance in computed tomography (CT) combined with magnetic resonance imaging or ultrasonography. The tumor size was estimated by the maximal diameter of the largest tumor of each kidney on CT. Tumors were classified as small $(<4$ $\mathrm{cm})$, medium $(4-10 \mathrm{~cm})$ or large $(>=10 \mathrm{~cm})$ based on the size. Selective transcatheter arterial embolization (TAE) was principally performed by the injection of both metallic coils and absolute ethanol following diagnostic arteriography. To evaluate the changes in tumor size after TAE, CT was repeated semiannually for 2 years and annually thereafter. For followup observation CT was annually repeated. Mean followup times for the whole period, during observation and after TAE in these patients were 60 (range 12-170), 52 (range 12-170) and 33 (range 12-78) months, respectively. Statistical analysis was performed using Mann Whitney-U analysis of variance by ranks to compare different groups.

\section{RESULTS}

The patients consisted of 6 males and 6 females with a mean age of 28.8 years (range 15-47). The diseases associated with TSC included mental retardation ( $n=7)$, lung lymphangiomyomatosis ( $\mathrm{n}=2)$, liver tumor $(\mathrm{n}=2)$ and retroperitoneal lymphangiomatosis $(n=1)$. Seven patients (58\%) presented with tumor-associated symptoms, including flank pain $(n=6)$ and a palpable mass $(n=1)$. A renal tumor was the first manifestation of TSC in 5 patients. In the remaining 5 patients (42\%) tumors were asymptomatic and incidentally discovered at evaluation for TSC $(n=4)$ and examination for polydipsia $(n=1)$. All but 1 patient had bilateral multiple tumors at initial presentation and that patient developed tumors in the contralateral kidney 2 years later. Thus, all patients had bilateral multiple tumors. The mean tumor size was $6.6 \mathrm{~cm}$ (range 1.5-15). Of the 24 tumors, 10, 6 and 8 tumors were classified as small, medium and large, respectively. Symptoms at presentation were noted in $0(0 \%), 2$ (33\%) and 6 (75\%) of the small, medium and large tumors, respectively. 
Initial and subsequent treatments are shown in Table 1. Among 14 kidneys (mean tumor size $4 \mathrm{~cm}$, range 1.5-12 cm) 8 tumors grew during the observation period. The percentages of growing lesions were $70 \%(7 / 10), 0 \%(0 / 2)$ and $50 \%(1 / 2)$ in small, medium and large tumors, respectively. Remarkable growth was observed in the third decade of life in 3 females and 1 male, but the growing speed did not exceed $1.5 \mathrm{~cm}$ per year. (Fig. 1). No patients experienced uncontrollable renal hemorrhage. Subsequent intervention was required in 4 patients for an abdominal mass or pain. Finally, a total of 3 nephrectomies and 11 embolizations were performed. Excluding 3 prophylactic interventions, $20 \%(2 / 10), 50 \%(2 / 4)$ and $100 \%(7 / 7)$ of small, medium and large tumors required intervention for symptoms, respectively.

All nephrectomies were done before 1991 and their indications were intractable flank pain in 2 kidneys and an abdominal mass in 1 kidney, whose tumors were multiple, large and consuming almost the entire kidney.

TAE has been a preferred intervention in our department since $1995 . \quad$ Indications for TAE were flank pain in 8 kidneys from 7 patients and prophylaxis in 3 kidneys whose tumor was asymptomatic but larger than $4 \mathrm{~cm}$. Reembolization was required in 5 kidneys because of tumor multiplicity $(n=4)$ and incompleteness due to vascular spasms $(n=1)$. Of the 8 symptomatic kidneys, clinical symptoms disappeared in 6 and did not change in 2 . All tumors decreased in size with a mean decrement rate of $43 \%$ (range $0-87$ ), excepting 1 tumor, which was $15 \mathrm{~cm}$ in size, composed of prominent fatty tissue and presented with a abdominal mass (Fig. 2). There was no significant statistical difference in the decrement rate between tumors $10 \mathrm{~cm}$ or more and those less than $10 \mathrm{~cm}(\mathrm{p}=0.12)$. In 15 of 16 procedures, flank pain and fever associated with postembolization syndrome occurred, but these symptoms could be reduced with conservative management. Renal atrophy occurred in 1 patient. Embolization was performed using 3 metallic coils and $17 \mathrm{ml}$ of absolute 
ethanol for a $10 \mathrm{~cm}$ tumor supplied by 2 feeding arteries from the 2nd renal arterial branch. He experienced severe postembolization syndrome. Followup CT obtained 3 months after TAE showed renal atrophy and his creatinine level had risen from 0.8 to $1.2 \mathrm{mg} / \mathrm{dl}$; however, he remained asymptomatic and normotensive. No tumors required further surgical treatment or drainage.

The mean reduction rates in renal function calculated by the reciprocal of serum creatinine after the intervention were 35\% (range 18-57) and 9\% (range 0-33) in patients after nephrectomy and TAE, respectively. At last followup 10 patients were alive without renal failure and 1 patient was lost to followup at 38 months.

\section{DISCUSSION}

TSC patients have a decreased life-expectancy in comparison with the general population. While central nervous disease is the commonest cause of death in childhood and adolescence, renal disease is in adulthood. ${ }^{2}$ Since neurologic care has improved recently, renal complications have become more prominent and long-term survival depends on their management. Renal lesions in TSC include cysts, AMLs and renal cell carcinomas. TSC is one of the phacomatoses and TSC patients are at risk for developing malignant tumors similar to other carcinoma-hamartoma syndromes. ${ }^{6,7}$ Although reports of renal malignancy in young TSC patients have suggested an association, renal cell carcinoma is, in fact, rare. Furthermore, recent advanced imaging techniques can differentiate AMLs from carcinomas and circumvent unnecessary nephrectomy. ${ }^{8} \quad$ Renal cysts are found in approximately $20 \%$ of patients. They usually develop in childhood and their incidence becomes stable with age. ${ }^{9} \quad$ The presence of numerous renal cysts can cause renal failure; however, its prevalence was reported to be $1-3 \% .^{10,11}$

AMLs occupy an important place in management of adult TSC patients, since 
AMLs are encountered in $60-80 \%$ of the patients, often develop bilaterally, and are large and symptomatic more frequently than sporadic AMLs. They develop in childhood, do not disappear and their incidence increases with age. ${ }^{9} \quad$ Dysmorphic vessels in AMLs can have microaneurysms or macroaneurysms. These aneurysms may rupture and bleed, resulting in significant morbidity: pain, loss of a renal unit, subsequent renal failure, and possibly death, although AMLs do not cause renal failure by themselves. ${ }^{10}$

Because of its benign nature, the principles of management are resolution of symptoms and the prevention of fatal morbidity without compromising renal function, except for rare cases of the malignant epithelial type. Although most AMLs can be managed conservatively, nephron-preserving intervention is recommended for tumors with symptoms and at the risk of hemorrhage. Total nephrectomy was performed earlier, but it should be, needless to say, avoided even if tumors extensively involve the entire kidney. Partial nephrectomy is a treatment option. Several authors reported excellent results as to the preservation of adequate renal function and tumor control; however, their studies included only a small number of TSC patients (3 of 55 patients). ${ }^{12,13}$ AMLs associated with TSC are unlikely to be amenable to partial nephrectomy because of their multiplicity.

TAE was performed to control acute hemorrhage temporarily, and has been used with increasing frequency to manage not only symptomatic tumors but also asymptomatic tumors at risk for hemorrhage because patient symptoms are usually the result of hemorrhage. TAE can effectively control symptoms in $80-93 \%$ of tumors and shrinks the majority of tumors. ${ }^{14}$ TAE is considered the first choice of intervention for symptomatic AMLs associated with TSC. The response to TAE is, however, related to the tumor's internal components. The angiomyogenic components nearly all disappear, but fatty components are slightly reduced after TAE. ${ }^{15}$ Reembolization, considered a disadvantage in TAE, was required in $45 \%$ of cases in this study, which was possibly attributable to the 
nature of tumors with multiplicity. We believe the repeatability of this intervention is an advantage in TSC patients. TAE is a safe and tolerable procedure, but a risk of unexpected large ischemic change should be kept in mind when treating a tumor with multiple feeding arteries. $^{16}$ To our knowledge there has been no randomized study on prophylactic intervention for AMLs, so long-term followup data on the natural course are needed to judge its efficacy.

The risk for hemorrhage from AMLs is unknown. Published series suggest that hemorrhage occurs in half of the patients and is related to the size of the tumor. ${ }^{17}$ Although the size cutoff point recommended by Oestering et al is $4 \mathrm{~cm}$, all tumors larger than $4 \mathrm{~cm}$ do not necessarily require intervention. As some authors reported that tumors larger than the 2nd cutoff point of $8 \mathrm{~cm}$ or $10 \mathrm{~cm}$ required intervention more frequently than small tumors, all lesions larger than $10 \mathrm{~cm}$ were considered to require intervention in this study. ${ }^{16,18}$ Furthermore, TSC patients were reported to have tumor enlargement and require intervention more frequently than sporadic patients. Steiner et al observed that $67 \%$ of AML grew and $50 \%$ required intervention. ${ }^{3}$ Van Baal et al reexamined 20 patients after an interval of 5 years and found that $20 \%$ had enlarged tumors, 35\% had renal hemorrhage requiring hospitalization, $10 \%$ required nephrectomy and 5\% died. ${ }^{4}$ These authors therefore recommended prophylactic intervention with semiannual followup for asymptomatic tumors larger than $4 \mathrm{~cm}$. Serendipitous rapid tumor growth, which exclusively occurred in the 2nd or 3rd decade with a maximal growth speed of $4 \mathrm{~cm}$ per year, was reported in TSC patients. $^{3,9,19}$ But these reports could not delineate the growth curve because of the long followup interval. The most interesting finding in this study was that the growth curve was linear even when they showed rapid growth. No patients had uncontrollable hemorrhage during followup until the lesion enlarged to $15 \mathrm{~cm}$ or presented symptoms.

These results suggest that immediate TAE is mandatory for symptomatic tumors 
and that annual followup is sufficient for asymptomatic tumors, especially for those showing slow growth or in patients after the 3rd decade. Patients with a tumor larger than $10 \mathrm{~cm}$ may be candidates for a prospective study for TAE. TAE is indicated when tumors present symptoms. Further acquisition of information on the natural history and molecular mechanism of the TSC gene and its products may provide information on the optimal management of renal AMLs associated with TSC.

\section{CONCLUSIONS}

Although asymptomatic AMLs associated with TSC gradually grow, severe hemorrhage is rare and most can be followed by annual computed tomography. Embolization is the first choice of intervention but should be reserved until when symptoms develop. Further followup study is warranted to optimize the management of renal AMLs associated with TSC. 


\section{REFERENCES}

1. Sampson, J.R. and Harris, P.C.: The molecular genetics of tuberous sclerosis. Human Mol Genet, 3: 1477, 1994.

2. Shepherd, C.W., Gomez, M.R. and Crowson, C.S.: Causes of death in patients with tuberous sclerosis. Mayo Clin Proc, 66: 792, 1991.

3. Steiner, M.S., Goldman, S.M., Fishman, E.K. and Marshall, F.F.: The natural history of renal angiomyolipoma. J Urol, 150: 1782, 1993.

4. Van Baal, J.G., Smits, N.J., Keeman, J.N., Lindhout, D. and Verhoef, S.: The evolution of renal angiomyolipomas in patients with tuberous sclerosis. J Urol, 152: 35, 1994.

5. Roach, E.S., Gomez, M.R. and Northrup H.: Tuberous sclerosis complex consensus conference: revised clinical diagnostic criteria. J Child Neurol, 13: 624, 1998.

6. Cook, J.A., Oliver, K., Mueller, R.F. and Sampson, J.: A cross sectional study of renal involvement in tuberous sclerosis. J Med Genet, 33: 480, 1996.

7. Al-Saleem, T., Wessner, L.L., Scheithauer, B.W., Patterson, K., Roach, E.S., Dreyer, S.J., et al: Malignant tumors of the kidney, brain, and soft tissues in children and young adults with the tuberous sclerosis complex. Cancer, 83: 2208, 1998.

8. Silverman, S.G., Pearson, G.D.N., Seltzer, S.E., Polger, M., Tempany, C.M.C., Adams, D.F., et al: Small ( $<$ or $=3 \mathrm{~cm}$ ) hyperechoic renal masses: Comparison of helical and conventional CT for diagnosing angiomyolipoma. AJR Am J Roentgenol, 167: 877, 1996.

9. Ewalt. D.H., Sheffield, E., Sparagana, S.P., Delgado, M.R. and Roach, E.S.: Renal lesion growth in children with tuberous sclerosis complex. J Urol, 160, 141, 1998.

10. Okada, R., Platt, M. and Fleishman, J.: Chronic renal failure in patients with tuberous sclerosis. Association with renal cysts. Nephron, 30: 85, 1982.

11. Schillinger, F. and Montagnac, R.: Chronic renal failure and its treatment in tuberous sclerosis. Nephrol Dial Transplant, 11: 481, 1996. 
12. Fazeli-Matin, S. and Novick, A.C.: Nephron-sparing surgery for renal angiomyolipoma. Urology, 52: 577, 1998.

13. Heidenreich, A., Hegele, A., Varga, Z., von Knobloch, R. and Hofmann, R.: Nephron-sparing surgery for renal angiomyolipoma. Eur Urol, 41: 267, 2002.

14. Mourikis, D., Chatziioannou, A., Antoniou, A., Kehagias, D., Gikas, D. and Vlahos, L.: Selective arterial embolization in the management of symptomatic renal angiomyolipomas. Eur J Radiol, 32: 153, 1999.

15. Han, Y.M., Kim, J.K., Roh, B.S., Song, H.Y., Lee, J.M., Lee, Y.H., et al: Renal angiomyolipoma: selective arterial embolization - effectiveness and changes in angiomyogenic components in long-term follow-up. Radiology, 204: 65, 1997.

16. Dickinson, M., Ruckle, H., Beaghler, M. and Hadley, H. R.: Renal angiomyolipoma: optimal treatment based on size and symptoms. Clin Nephrol, 49: 281, 1998.

17. Oestering, J.E., Fishman, E.K., Goldman, S.M. and Marshall, F.F.: The management of renal angiomyolipoma. J Urol, 135: 1121, 1986.

18. Kennelly, M.J., Grossman, H.B. and Cho, K.J.: Outcome analysis of 42 cases of renal angiomyolipoma. J Urol, 152: 1988, 1994.

19. Lemaitre, L., Robert, Y., Dubrulle, F., Claudon, M., Duhamel, A., Danjou, P., et al: Renal angiomyolipoma: growth followed up with CT and/or US. Radiology, 197: 598, 1995. 
Table 1 Treatment in each tumor size group

Treatment

Initial tumor size

small medium large

Initially asymptomatic tumor

Observation continued

Subsequent intervention required after observation

Prophylactic intervention performed

Initially symptomatic tumor

Subsequent intervention required after observation

Immediate intervention performed

$8 \quad 2 \quad 0$

$2 \quad 0 \quad 1$

$\begin{array}{lll}0 & 2 & 1\end{array}$

Totals

10

6

$8(3)$

Numbers in parentheses indicate the number of nephrectomies. 


\section{FIGURE LEGENDS}

Figure 1 Tumor size according to age. Tumor size examined at first presentation and during followup is plotted against age. Crosses indicate that intervention was performed.

Figure 2 Tumor size according to time after TAE. Asterisks indicate that reembolization was performed. 
Figure 1

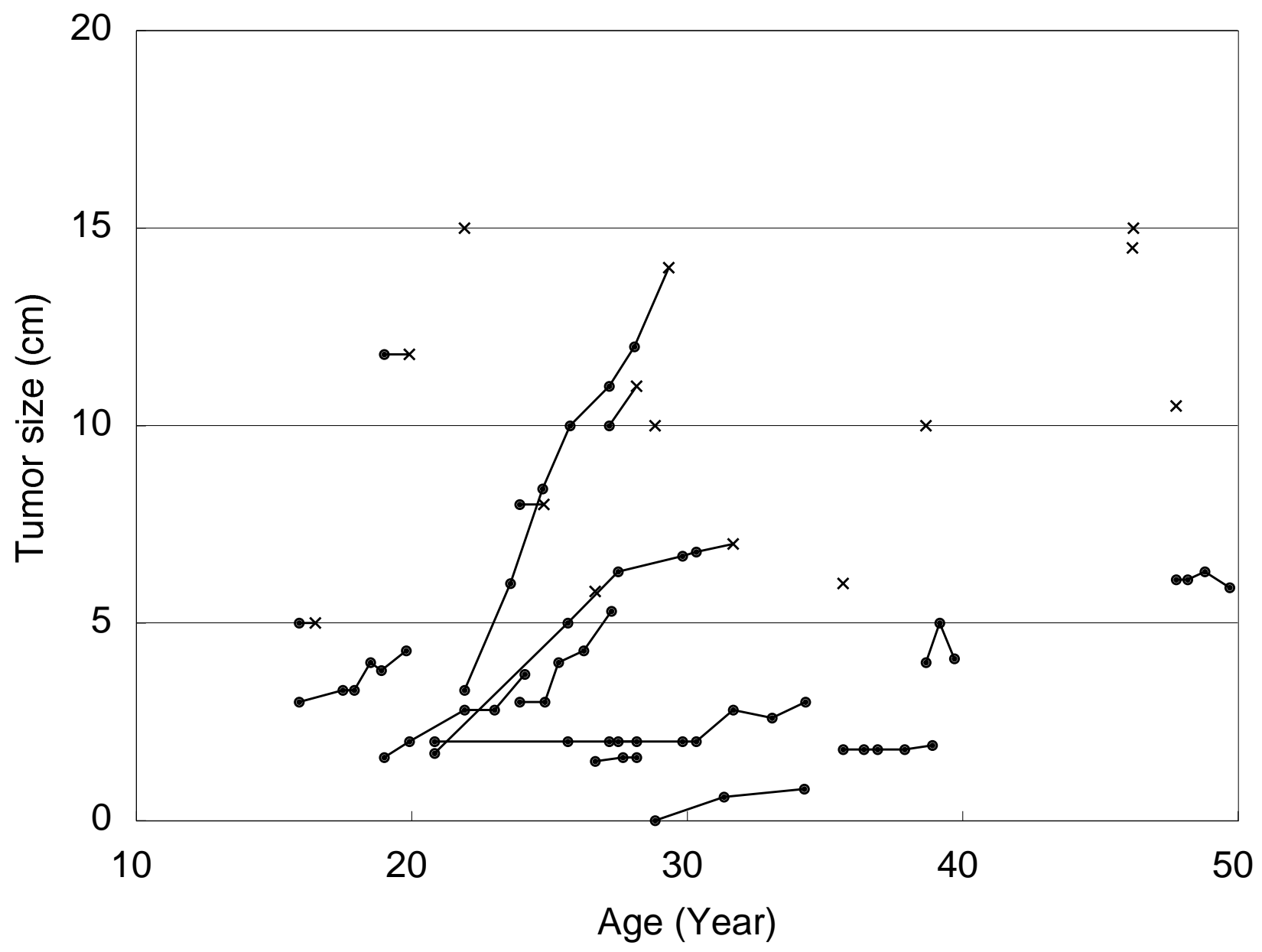


Figure 2. Tumor Size after Embolization

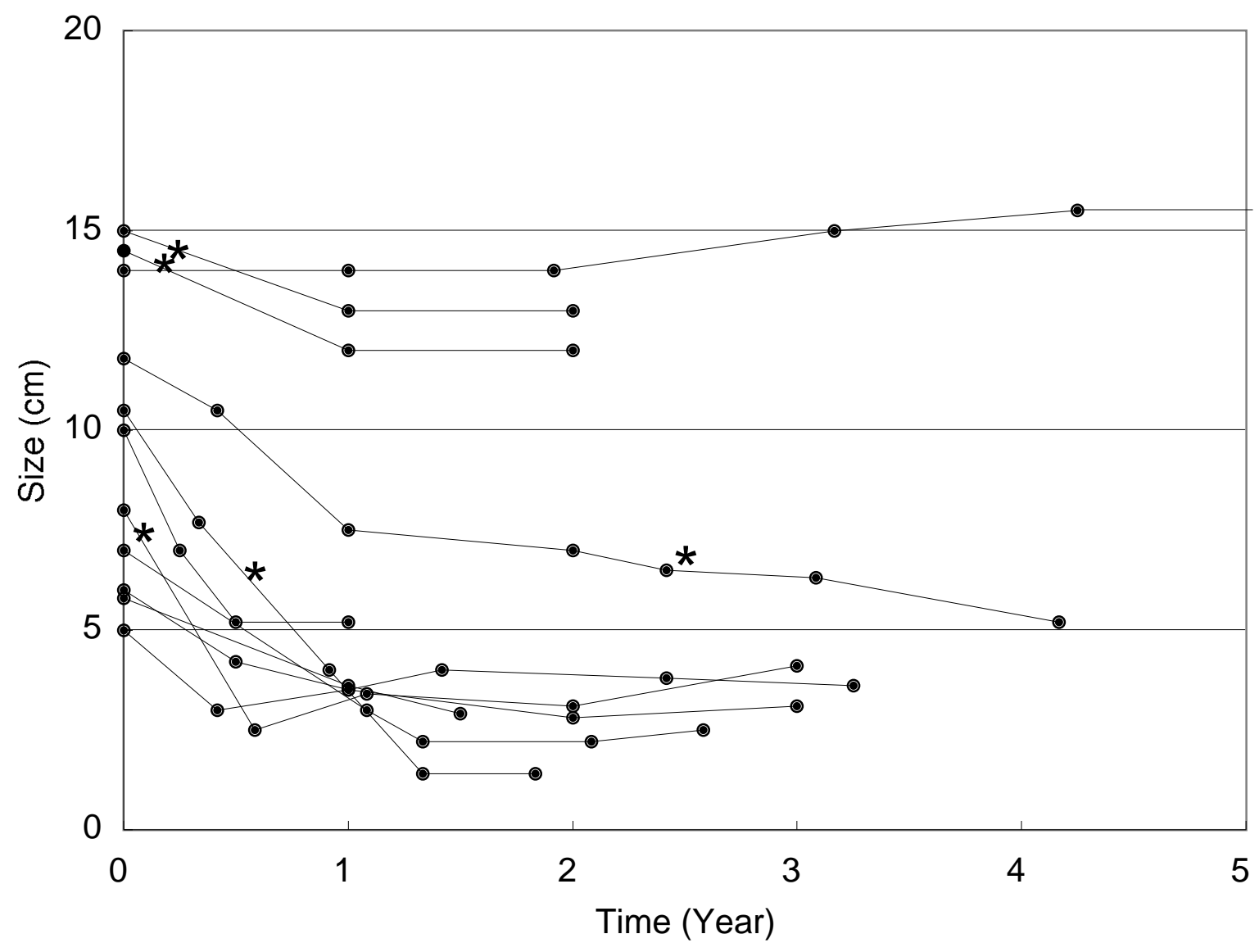

